05

\title{
Формирование линии сигнала ядерного магнитного резонанса в слабом поле
}

\author{
(C) В.В. Давыдов ${ }^{1,2,3}$, Н.С. Мязин ${ }^{1}$, В.Б. Фадеенко ${ }^{1}$, С.Э. Логунов ${ }^{1}$ \\ ${ }^{1}$ Санкт-Петербургский политехнический университет Петра Великого, \\ Санкт-Петербург, Россия \\ ${ }^{2}$ Санкт-Петербургский государственный университет телекоммуникаций \\ им. профр. М.А. Бонч-Бруевича, Санкт-Петербург, Россия \\ ${ }^{3}$ Всероссийский научно-исследовательский институт фиитопатологии, \\ Большие Вяземы, Московская обл., Россия \\ E-mail: Davydov_vadim66@mail.ru
}

Поступило в Редакцию 25 октября 2017 г.

Предложена новая модель описания структуры линии сигнала ядерного магнитного резонанса, регистрируемого в слабом магнитном поле от конденсированной среды. Представлены результаты исследований формы линии сигнала ядерного магнитного резонанса, регистрируемого с использованием модуляционной методики в слабом магнитном поле от малого объема конденсированной среды. Разработана новая методика определения в экспресс-режиме состава смеси конденсированной среды и относительных концентраций ее компонентов.

DOI: 10.21883/PJTF.2018.04.45638.17096

В настоящее время одной из актуальных задач прикладной физики является разработка быстрых и надежных методов экспресс-контроля состояния конденсированных сред [1-4]. Одно из наиболее перспективных решений данной задачи - использование явления ядерного магнитного резонанса (ЯМР) [1,3-6]. Проведенные нами исследования, а также опыт эксплуатации приборов для экспресс-контроля параметров конденсированных сред показали, что другие методы, используемые для решения задач контроля параметров среды в экспресс-режиме, по сравнению с ЯМР обладают рядом существенных недостатков [1,3-6]. Они менее информативны по числу измеряемых параметров среды, предназначены в основном для тестирования только небольшого числа сред (например, воды, жиров или моторного масла) и т.д. Кроме того, проведенные методом ЯМР исследования не вносят необратимых изменений в физическую структуру и химический состав среды в отличие от 
других методов. При исследовании состояния конденсированной среды в экспресс-режиме методом ЯМР в слабом магнитном поле сигнал от нее регистрируется с использованием модуляционной методики $[1,4,6]$. Другие способы регистрации сигнала ЯМР в слабом магнитном поле не позволяют обеспечить необходимую погрешность измерения (не более $1 \%$ ) для получения достоверных результатов при проведении исследований $[4,6]$.

Одним из недостатков использования модуляционной методики при исследовании конденсированных сред в экспресс-режиме является отсутствие теоретической модели, позволяющей воспроизводить форму линии регистрируемого сигнала ЯМР в слабом поле и определять ее структуру. Полученные нами ранее экспериментальные результаты показывают, что это существенно ограничивает возможности исследования конденсированных сред в экспресс-режиме методом ЯМР. Поэтому цель настоящей работы состоит в разработке теоретической модели, позволяющей воспроизводить форму линии регистрируемого сигнала ЯМР в условиях модуляции слабого магнитного поля в межполюсном пространстве малогабаритного ЯМР-спектрометра.

Движение продольных и поперечных компонент вектора намагниченности конденсированной среды в катушке регистрации ЯМР-спектрометра описывается уравнениями Блоха [7-9]. В работах [9-12] представлены различные аналитические решения уравнений Блоха в зависимости от условий прохождения резонанса. Поскольку в стационарных ЯМР-спектрометрах в основном используется линейное изменение магнитного поля $H_{0}$, данному случаю модуляции во многих работах [9-12] уделено более пристальное внимание, чем другим. При линейной модуляции поля $H_{0}$ из уравнений Блоха для регистрируемых спектрометром сигналов поглощения $v(t)$ и дисперсии $u(t)$ были получены следующие соотношения [9-11]:

$$
\begin{gathered}
v(t)=v_{0} \exp \left(-t / T_{2}^{*}\right) \cos \left(a t^{2} / 2\right), \\
u(t)=u_{0} \exp \left(-t / T_{2}^{*}\right) \sin \left(a t^{2} / 2\right),
\end{gathered}
$$

где $a=\gamma \frac{d H_{z}}{d t}=\frac{d \Delta \omega}{d t} \quad$ скорость изменения расстройки магнитного поля, $\gamma$ - гиромагнитное отношение ядра, $T_{2}^{*}$ - эффективное время поперечной релаксации, $v_{0}$ и $u_{0}$ - максимальные значения амплитуд регистрируемого сигнала поглощения и дисперсии ЯМР.

$4^{*}$ Письма в ЖТФ, 2018, том 44, вып. 4 
В работах [9-12] для различных сред, находящихся в стационарном состоянии, приведены примеры зарегистрированных сигналов ЯМР (поглощения и дисперсии) и их расчетные зависимости, полученные с помощью формул (1) и (2), а также показано соответствие теории эксперименту. При сравнении сигналов ЯМР, зарегистрированных с использованием модуляционной методики от воды, находящейся как в стационарном, так и в текущем состоянии, с расчетами, выполненными с помощью (1) и (2) при линейном изменении $H_{0}$, также наблюдается их внешнее сходство. На рис. 1 в качестве примера представлены данные зависимости при $T=291.6 \mathrm{~K}$.

Анализ представленных на рис. 1 зависимостей показывает, что форма линии сигналов ЯМР при определенных параметрах модуляции поля $H_{0}$ представляет собой набор пиков („виглей“), амплитуды которых со временем затухают по экспоненте. По этой причине в работах [5,11-13] для исследования различных сред, находящихся как в стационарном, так и в текущем состоянии, с помощью сигналов ЯМР, регистрируемых с использованием модуляционной методики, был предложен способ определения времени поперечной релаксации $T_{2}$ по спаду огибающей, которая аппроксимируется зависимостью $\exp \left(-t / T_{2}^{*}\right)$. Значения $T_{2}$, полученные для различных сред с использованием данного способа, совпали в пределах погрешности измерения со значениями $T_{2}$, измеренными на других приборах. Поэтому авторами работ $[5,11-13]$ был сделан вывод, что в случае необходимости для описания формы линии регистрируемого с использованием модуляционной методики сигнала ЯМР можно применять соотношения (1) и (2). Дальнейшие исследования ими не проводились.

В слабом магнитном поле для обеспечения регистрации сигнала ЯМР от различных ядер, обладающих магнитным моментом, значение поля $H_{1}$ автодинного детектора устанавливается по максимуму отношения сигнал/шум $(S / N)[1,4,6]$. В этом случае регистрируемый автодинным детектором сигнал ЯМР от конденсированной среды является суммой сигналов поглощения и дисперсии. Кроме того, в [6] нами были установлены соотношения между значениями $H_{1}$, амплитудой $H_{m}$ и частотой $f_{m}$ поля модуляции $H_{0}$, при выполнении которых в малогабаритном ЯМР-спектрометре реализуется регистрация сигнала ЯМР.

Проведенные нами эксперименты показали, что ни при каких значениях $H_{1}, H_{m}$ и $f_{m}$, удовлетворяющих соотношениям в [6], обеспечить

Письма в ЖТФ, 2018, том 44, вып. 4 

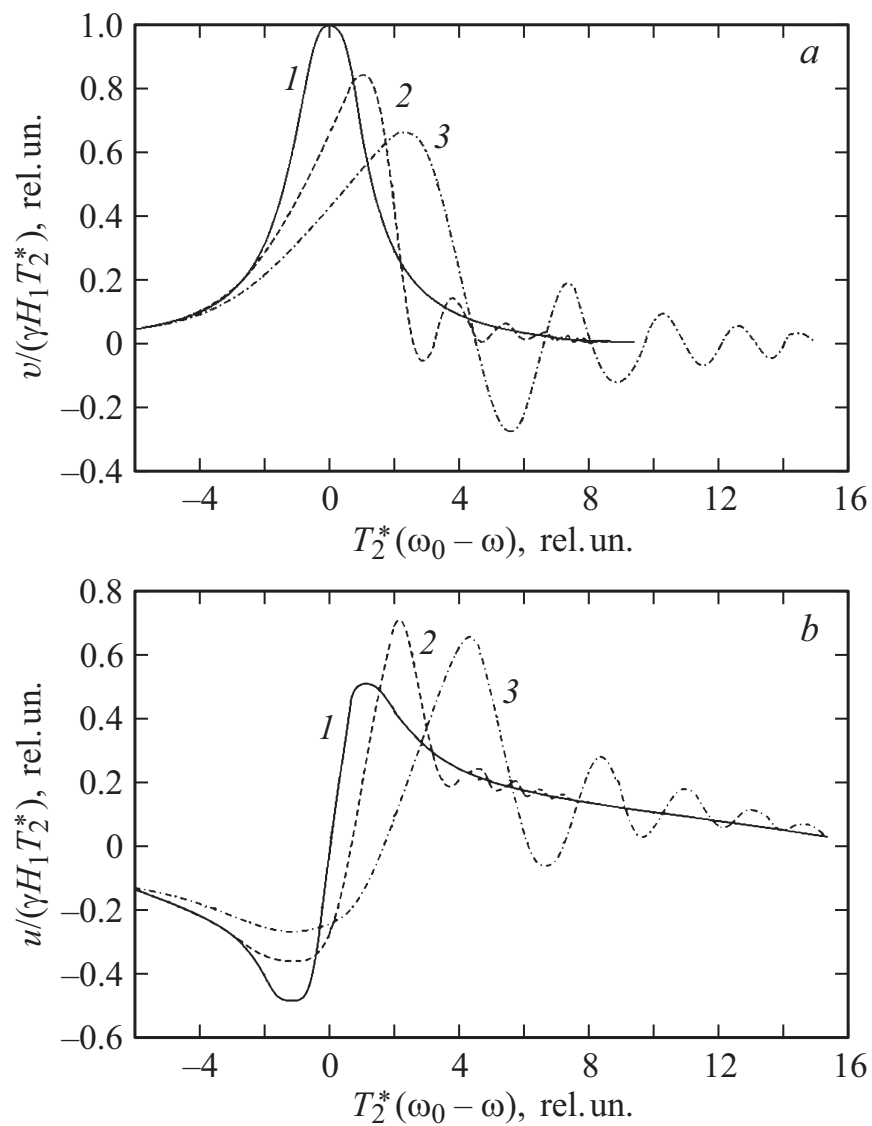

Рис. 1. Сигналы ЯМР от воды: $a$ и $b-$ расчет с использованием формул (1) и (2), кривым соответствуют значения $d^{1 / 2} T_{2}^{*}=0(1), 1$ (2) и $2(3) ; c-$ сигнал ЯМР, зарегистрированный в слабом поле $\left(B_{0}=0.082 \mathrm{~T}\right)$ с использованием модуляционной методики.

при регистрации сигнала ЯМР условие быстрого адиабатического прохождения через резонанс, при котором были получены (1) и (2), в малогабаритном ЯМР-спектрометре невозможно. Поэтому нами в уравнениях Блоха было предложено учитывать использование модуля-

Письма в ЖТФ, 2018, том 44, вып. 4 


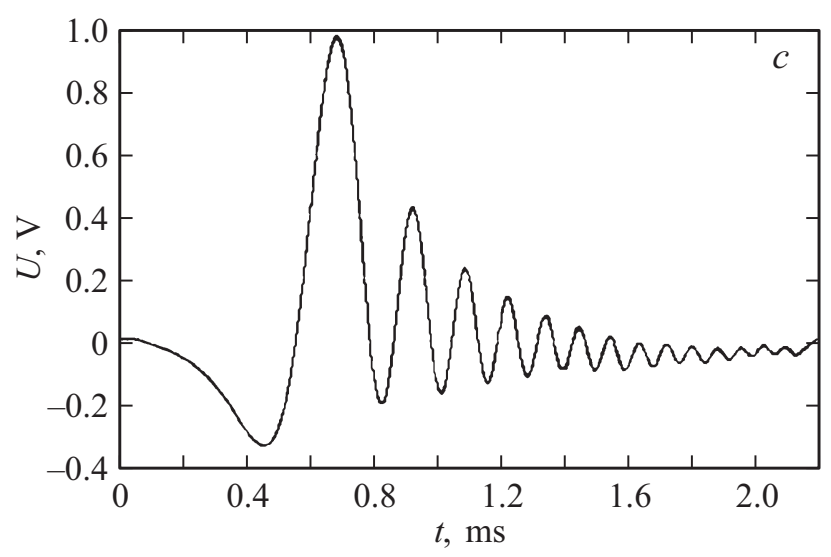

Рис. 1 (продолжение).

ционной методики в изменении магнитного поля $H$ между полюсами магнитов следующим образом:

$$
H=H_{0}+H_{m} \sin \left(\omega_{m} t\right)
$$

где $H_{0}-$ постоянное магнитное поле, $H_{m}$ и $\omega_{m}-$ амплитуда и частота поля модуляции.

В этом случае изменение расстройки частоты поля от резонанса в системе уравнений Блоха [7-10] будет иметь следующую зависимость:

$$
\Delta \omega=\gamma H_{0}+\gamma H_{m} \sin \left(\omega_{m} t\right)-\omega_{n m r} .
$$

Одной из особенностей регистрации сигнала ЯМР в слабом магнитном поле с использованием модуляционной методики является то, что она должна осуществляться только на частоте резонанса $\left(\omega_{n m r}=\omega_{0}=\gamma H_{0}\right)$. В этом случае в уравнениях Блоха в соответствии c (4) необходимо использовать соотношение

$$
\Delta \omega=\gamma H_{m} \sin \left(\omega_{m} t\right)
$$

В этом случае система уравнений Блоха во вращающейся системе координат в переменных $v(t), u(t)$ и $M_{z}(t)$ с учетом (5) принимает

Письма в ЖТФ, 2018, том 44, вып. 4 


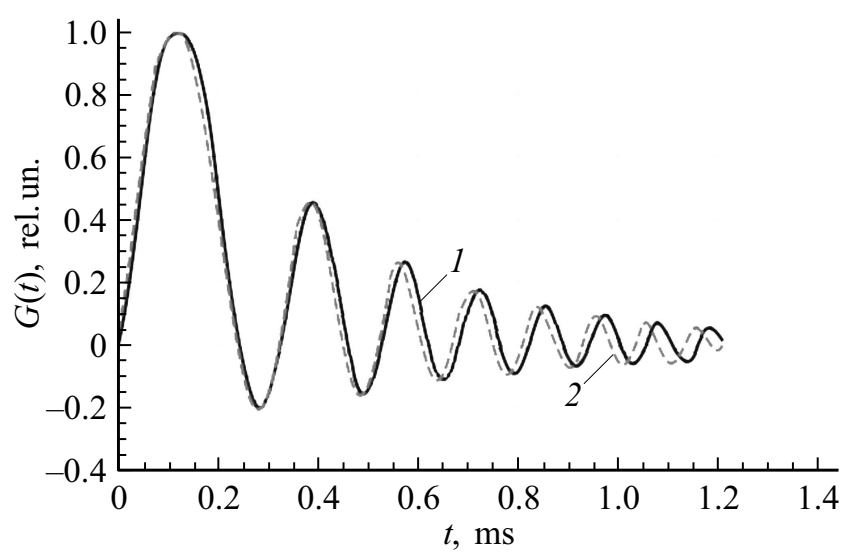

Рис. 2. Форма линии сигнала ЯМР от водопроводной воды. Кривая 1 соответствует эксперименту, 2 - расчету.

следующий вид:

$$
\begin{gathered}
d u(t) / d t+u(t) / T_{2}+\gamma H_{m} \sin \left(\omega_{m} t\right) v(t)=0, \\
d v(t) / d t+v(t) / T_{2}-\gamma H_{m} \sin \left(\omega_{m} t\right) u(t)+\gamma H_{1} M_{z}(t)=0, \\
d M_{z}(t) / d t+M_{z}(t) / T_{1}-\chi_{0}\left(H_{0}+H_{m} \sin \left(\omega_{m} t\right)\right) / T_{1}-\gamma H_{1} v(t)=0,
\end{gathered}
$$

где $\chi_{0}-$ статистическая ядерная магнитная восприимчивость, $T_{1}-$ время продольной релаксации.

Система уравнений (6) решается относительно компонент $v(t), u(t)$ и $M_{z}(t)$ с учетом начальных условий $M_{z}(0)=\chi_{0} H_{0}, v(0)=0, u(0)=0$. Поскольку автодинный детектор, который используется для регистрации сигналов ЯМР в слабом магнитном поле, является интегральным измерительным устройством, форма линии $G(t)$ регистрируемого сигнала ЯМР от конденсированной среды описывается следующим соотношением:

$$
G(t)=\left(A v^{2}(t)+B u^{2}(t)\right)^{1 / 2},
$$

где $A, B$ - коэффициенты, учитывающие вклад сигналов поглощения и дисперсии.

Письма в ЖТФ, 2018, том 44, вып. 4 


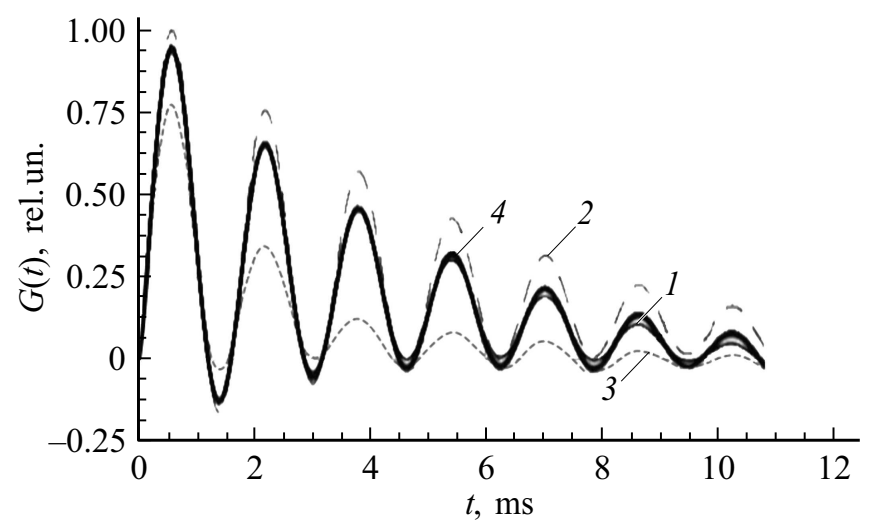

Рис. 3. Формы линий сигналов ЯМР. 1 - экспериментальный сигнал от смеси бензинов АИ-95 и А-76 в пропорции $75 \%$ к 25\%, 2-4 - результаты расчета сигналов ЯМР от чистых бензинов АИ-95 (2) и А-76 (3), от смеси бензинов АИ-95 и А-76 в пропорции $75 \%$ к $25 \%$ (4).

На рис. 2 в качестве примера представлены результаты сравнения формы пиков („виглей“) в линии регистрируемого сигнала ЯМР с расчетом их формы в ней, выполненным с использованием (7) с учетом особенностей регистрации сигнала ЯМР автодинным детектором при ее построении. В качестве конденсированной среды использовалась водопроводная вода при $T=293.1 \mathrm{~K}$. Для расчета $G(t)$ численные значения сигналов поглощения и дисперсии $v(t)$ и $u(t)$ были получены из решения системы уравнений (6).

Анализ представленных на рис. 2 зависимостей показывает, что предложенная нами модель с использованием решений уравнений Блоха с новыми коэффициентами (6) позволяет воспроизводить форму линии сигнала ЯМР, регистрируемого с помощью модуляционной методики, с погрешностью не более $3 \%$ (до пятого пика), а также впервые дает возможность определять вклад сигналов поглощения и дисперсии в сигнал ЯМР, регистрируемый в экспресс-режиме от конденсированной среды в слабом магнитном поле.

Это позволило нам разработать новую методику моделирования формы линии регистрируемого сигнала ЯМР от смеси, образованной несколькими средами, которые не вступили между собой в химическую

Письма в ЖТФ, 2018, том 44, вып. 4 
реакцию, а образовали конгломерат (например, смесь двух бензинов или бензин и керосин и т.д.). В этом случае регистрируемый сигнал ЯМР от такой смеси представляет собой сумму сигналов ЯМР от каждого из ее компонентов, и его форму линии $G(t)$ можно представить соотношением

$$
\begin{gathered}
G(t)=\sqrt{A_{m} v_{m}^{2}(t)+B_{m} u_{m}^{2}(t)}=\sum_{i=1}^{k} V_{i} \cdot N_{i} \sqrt{A_{i} v_{i}^{2}(t)+B_{i} u_{i}^{2}(t)}, \\
\sum_{i=1}^{k} V_{i}=V_{r},
\end{gathered}
$$

где $A, B-$ коэффициенты, определяющие вклад сигналов поглощения и дисперсии в сигнал ЯМР ( $m$ - смесь, $i-$ компоненты смеси $), N_{i}-$ число протонов в единице объема для сред, образующих смесь, $V_{i}-$ объем компонента смеси, $V_{r}$ - объем катушки регистрации.

На рис. 3 в качестве примера представлены результаты сравнения формы пиков („виглей“) зарегистрированного сигнала ЯМР от смеси бензинов АИ-95 и А-76 в пропорции 75\% к 25\% с расчетами формы пиков в линиях сигналов ЯМР от бензинов АИ-95 и А-76, а также от их смеси в отмеченной выше пропорции.

Анализ полученных результатов показывает, что расчетная форма линии сигнала ЯМР от смеси двух бензинов совпадает с экспериментальной зависимостью с погрешностью не более $3 \%$ (до пятого пика). Кроме того, рассчитанные с использованием (8) и (9) концентрации бензинов АИ-95 и А-76 в исследуемой смеси совпали в пределах погрешности измерений с их относительными концентрациями, которые были использованы для приготовления из них смеси перед экспериментом. Все это подтверждает достоверность предложенного нами метода.

\section{Список литературы}

[1] Karseev A.Yu., Vologdin V.A., Davydov V.V. // J. Phys.: Conf. Ser. 2015. V. 643. N 1. P. 012108.

[2] Васильев Н.С., Есаков А.А., Голяк Ил.С., Голяк Иг.С., Морозов А.Н., Табалин С.E. // ПТЭ. 2015. № 1. С. 181-182.

[3] Neronov Y.I., Karshenboim S.G. // Phys. Lett. A. 2003. V. 318, N 1-2. P. 126132.

Письма в ЖТФ, 2018, том 44, вып. 4 
[4] Давыдов В.В., Мязин Н.С. // Измерительная техника. 2017. № 2. С. 58-62.

[5] Дудкин В.И., Пахомов Л.Н. Квантовая электроника. СПб.: СПбПУ, 2012. $496 \mathrm{c}$.

[6] Давыдов В.В., Мязин Н.С., Величко Е.Н. // Письма в ЖТФ. 2017. Т. 43. B. 13. C. $34-42$.

[7] Bloch F. // Phys. Rev. 1946. V. 70. N 7. P 460-478.

[8] Bloch F., Hansen W.W., Packard F. // Phys. Rev. 1946. V. 70. N 7. P. 474-492.

[9] Абрагам А. Ядерный магнетизм. М.: ИЛ, 1967. 686 с.

[10] Леше А. Ядерная индукция. М.: ИЛ, 1963. 684 с.

[11] Бородин П.М., Вологдин М.И, Москалев В.В., Морозов А.А. Ядерный магнитный резонанс. Л.: Изд-во ЛГУ, 1982. 344 с.

[12] Чижсик В.И. Ядерная магнитная релаксация. Л.: Изд-во ЛГУ, 1991. 256 с.

[13] Жерновой А.И. Ядерно-магнитные расходомеры. Л.: Машиностроение, 1985. $136 \mathrm{c}$ 